\title{
This Month in AJP
}

\section{Battling Contact Dermatitis at the Local Level}

Keratinocytes express the cortisone-activating enzyme $11 \beta$ hydroxysteroid dehydrogenase 1 (11 $\beta$-HSD1), which regulates proliferation as well as inflammation. To clarify the enzyme's function in keratinocytes, Terao et al (Am J Pathol 2016, 186:1499-1510) analyzed the inflammatory response in 11 $\beta$-HSD1-knockout mice to hapten-induced contact dermatitis. Mice displayed enhanced ear swelling and increased inflammatory cell infiltration following various low-dose chemical-induced irritant dermatitis protocols; the reaction was dose-dependently suppressed by topical corticosterone. In humans, $11 \beta$-HSD1 expression was decreased in the epidermis of lesional psoriasis vulgaris skin compared with healthy skin. Directing treatment at the local cortisol activation system in keratinocytes represents a novel pharmaceutical strategy for skin inflammation.

\section{Improving Pancreatic Cancer Models}

Commercially available, highly passaged pancreatic cancer cell lines offer poor predictive value in clinical studies, presenting a major barrier to the development of new therapies. Pham et al (Am J Pathol 2016, 186:1537-1546) therefore established a primary pancreatic cancer cell line (PPCL) from patient-derived xenografts. PPCLs were highly reproducible and biologically stable when passaged in vitro as determined by both morphology and tumorigenicity. Pancreatic tumor characteristics and markers were retained after grafting, isolation, culture, and then reintroduction into mice. These patient-derived PPCLs will enable better preclinical modeling and subsequent treatment of pancreatic cancer.

\section{Myostatin Inhibition in Nemaline Myopathies}

Myofiber smallness (or hypotrophy) may contribute to weakness in nemaline myopathies (NMs), a group of congenital muscle diseases characterized by nemaline rods identified by muscle biopsy. Using an established mouse model of NM, Tinklenberg et al (Am J Pathol 2016, 186:1568-1581) induced myofiber hypertrophy to explore its symptomatic benefit. Treatment with the myostatin signaling inhibitor ActRIIB-mFc significantly increased body mass, muscle mass, and quadriceps myofiber size and improved survival in mice.
However, it did not measurably increase limb strength. The mortality in male NM mice resulted from complications of urethral obstruction, which was subverted by ActRIIB-mFc treatment. These data identify a new phenotype of weakness possibly affecting survival in mouse models of neuromuscular disease.

\section{HU177 Epitope Regulates Ovarian Tumor Growth}

The use of monoclonal antibody (D93/TRC093) against the cryptic collagen epitope HU177 has shown promising results in phase 1 human clinical trials in patients with advanced malignant tumors, especially ovarian cancer. Caron et al (Am J Pathol 2016, 186:1649-1661) studied HU177 in ovarian cancer and found that HU177 is substantially expressed on $\alpha$-smooth muscle actin ( $\alpha$ SMA)-expressing stromal cells. Blocking HU177 with anti-HU177 antibody inhibited growth and proliferation of tumors generated by an ovarian cancer cell line and reduced the accumulation of $\alpha_{\mathrm{SMA}^{+}}{ }^{+}$cells in tumors as well as their adhesion and migration on denatured collagen. $\alpha_{10} \beta_{1}$ integrin served as a receptor for the HU177 epitope, and blocking this interaction decreased fibroblast migration. These data provide mechanistic insights in how the HU177 epitope regulates ovarian tumor growth.

\section{ACE2 Overexpression Reverses Diabetic Retinopathy}

Renin angiotensin system (RAS) inhibitors show unimpressive clinical outcomes for diabetic retinopathy, though successful in established animal models. Dominguez et al (Am J Pathol 2016, 186:1688-1700) assessed whether local retinal overexpression of a critical RAS componentangiotensin-converting enzyme (ACE)-2-could prevent or reverse diabetic retinopathy in streptozotocin-induced diabetic mice. Local overexpression of ACE2 prevented and partially reversed diabetic increases of acellular capillaries and infiltrating inflammatory cells into the retina as well as leukostasis. Locally restoring the balance between the classic and vasoprotective renin angiotensin system may reduce or even prevent vascular complications from diabetes. 